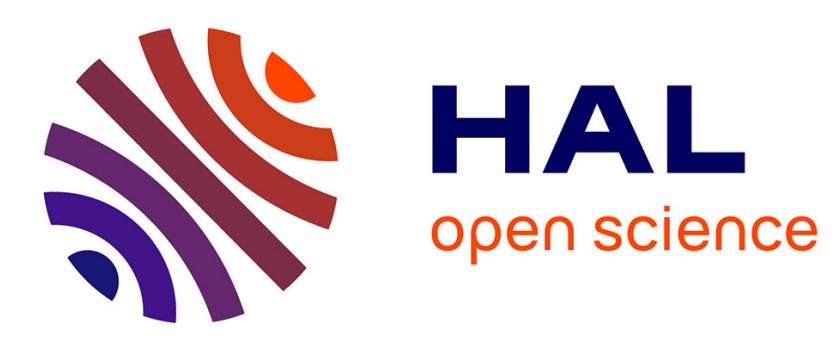

\title{
Intelligence Artificielle, ontologies et connaissances en médecine. Les limites de la mécanisation de la pensée
} Gunnar Declerck, Jean Charlet

\section{To cite this version:}

Gunnar Declerck, Jean Charlet. Intelligence Artificielle, ontologies et connaissances en médecine. Les limites de la mécanisation de la pensée. Revue des Sciences et Technologies de l'Information - Série RIA : Revue d'Intelligence Artificielle, 2011, 25 (4), pp.445-472. inserm-00916651

\section{HAL Id: inserm-00916651 https://www.hal.inserm.fr/inserm-00916651}

Submitted on 10 Dec 2013

HAL is a multi-disciplinary open access archive for the deposit and dissemination of scientific research documents, whether they are published or not. The documents may come from teaching and research institutions in France or abroad, or from public or private research centers.
L'archive ouverte pluridisciplinaire HAL, est destinée au dépôt et à la diffusion de documents scientifiques de niveau recherche, publiés ou non, émanant des établissements d'enseignement et de recherche français ou étrangers, des laboratoires publics ou privés. 


\title{
Intelligence artificielle, ontologies et connaissances en médecine
}

\section{Les limites de la mécanisation de la pensée}

\author{
Gunnar Declerck - Jean Charlet \\ Laboratoire de Santé Publique et Informatique Médicale, INSERM \\ Centre de recherche des Cordeliers \\ 15, rue de l'école de médecine, 75006 Paris \\ gunnar.declerck@crc.jussieu.fr
}

\begin{abstract}
RÉSUMÉ. Cet article de tonalité théorique vise à dresser un état des lieux des dernières avancées en ingénierie des connaissances médicales dans le domaine spécifique de la conception d'ontologies et systèmes à base de connaissances. Reprenant des débats ayant animé le paysage de l'intelligence artificielle (IA) à partir des années 1960, sous l'impulsion des travaux de H. L. Dreyfus, il vise notamment à montrer que la plupart des difficultés aujourd'hui rencontrées par l'ingénierie des connaissances dans le domaine de la santé sont inhérentes à la nature même de l'IA, en tant que projet de mécanisation de l'activité cognitive. Et il promeut à ce titre l'idée que seule une juste compréhension de ce que les machines ne peuvent faire, étant donné leur caractère machinique même, et qui reste, malgré sa finitude cognitive, une propriété exclusive de l'humain, pourra offrir d'équilibrer la balance entre les tâches allouables aux machines et celles laissées à la charge de ce dernier.

ABSTRACT. This theoretical article aims to draw up an inventory of the latest advances in medical knowledge engineering in the specific area of ontologies and knowledge based systems design. Echoing the debates that animated the landscape of Artificial Intelligence (AI) from the 1970s under the impetus of Dreyfus HL, it aims to show that most of the difficulties currently faced by medical knowledge engineering are inherent in the nature of AI, whose project is the mechanization of cognitive activity. As such it promotes the idea that only a fair understanding of what machines can do, given their machinic character itself, and remains, despite its cognitive finitude, a property of human being, may offer to balancing the scales between tasks that can be allocated to machines and those that have to be left in charge of humans.

MOTS-CLÉS: épistémologie de l'intelligence artificielle, ontologies, systèmes à base de connaissances, raisonnement médical, formalisation des connaissances.

KEYWORDS: epistemology of artificial intelligence, ontologies, knowledge-based systems, medical reasoning, knowledge formalization.
\end{abstract}

DOI:10.3166/RIA.25.445-472 @ 2011 Lavoisier, Paris

RSTI - RIA - 25/2011. Intelligence artificielle et santé, p. 445 à 472 


\section{Introduction. Ontologies et désorientation}

Dans le domaine de la santé, les ontologies sont principalement utilisées pour normaliser le codage des informations, que ce soit lors de la phase de rédaction des documents, ou lors d'un traitement postérieur destiné à leur conférer un format les rendant exploitables pour des traitements automatiques. Elles ont en ce sens un rôle normatif analogue aux terminologies médicales classiques (notamment les thésaurus) : mettre en place un vocabulaire commun et faire usage de représentations et concepts partagés, afin de permettre l'interopérabilité des documents, et l'élaboration de bases de connaissances médicales pourquoi pas universelles, dépassant les particularités régionales et linguistiques de chacun. Toutefois, leur approche formelle de la sémantique les distingue également clairement des systèmes terminologiques traditionnels : les ontologies sont des architectures de concepts, non des listes organisées de termes. Les concepts, à la différence des termes, se caractérisent par des définitions formelles, ce qui les rend manipulables par des machines. Faire usage d'une ontologie pour normaliser un document médical, c'est en ce sens encoder l'information en lui conférant un caractère qui la rend immédiatement manipulable par des machines, qui vont pouvoir opérer différents raisonnements exploitant les propriétés formelles des concepts. Les ontologies peuvent ainsi être utilisées pour des applications plus complexes que la seule normalisation des données, comme l'indexation automatique de documents ${ }^{1}$, la fouille de données pour l'extraction de connaissances et la recherche d'informations ${ }^{2}$, le clustering (procédé visant à regrouper des documents ou données suivant des critères préalablement définis), ou encore la structuration de bases de connaissances exploitées par des programmes d'aide au diagnostic ${ }^{3}$.

Une des principales fonctions des ontologies et systèmes à base de connaissances (SBC) en médecine est de combattre la désorientation. Avec le développement des machines numériques, c'est un nouveau support de mémoire qui s'est imposé, beaucoup plus puissant et beaucoup moins onéreux que son prédécesseur, le papier. Le numérique permet de stocker une quantité quasi infinie de données. Mais du fait même de cette possibilité, les données stockées n'ont plus besoin de faire l'objet d'un filtrage en amont qui n'autoriserait le stockage que des données pertinentes (méritant donc d'être enregistrées). Puisque les machines ont suffisamment de mémoire pour tout stocker, puisque cela ne coûte quasiment rien, stockons tout, nous ferons le tri après. Aussi cette possibilité fait-elle aussitôt naitre un problème : celui de s'orienter dans ces données, celui de l'accès et de la sélection des données pertinentes, celui du recoupement et de la synthèse : en bref le problème d'exploiter ces données pour générer des connaissances, c'est-à-dire des expectatives, schémas, modélisations ou hypothèses sur la réalité qui vont permettre d'aiguiller l'action et la

1. Cf. Mazuel \& Charlet (2009).

2. Cf. Rector et al. (1999).

3. Cf. Douali \& Jaulent (2011). 
prise de décision ${ }^{4}$. Ce problème n'était certes pas absent avec le stockage des connaissances et données sur le papier, mais il était alors beaucoup moins impérieux en raison des contraintes inhérentes à cette forme d'inscription : nécessitant des dispositifs parfois lourds pour sa duplication, n'ayant pas un coût nul, présentant un encombrement de l'espace important, et un format rendant parfois difficile la recherche d'informations - autant de raisons poussant à filtrer par avance les données allant être stockées, en fonction de leur pertinence.

Le problème de la désorientation se pose de manière on ne peut plus concrète dans le domaine de la médecine. Le personnel de santé de tous les points du globe peut bien consigner ses observations dans les mémoires de serveurs accessibles de partout quasiment sans délai, si l'on ne dispose pas de moyens de normaliser, filtrer et synthétiser ces données, ou avant même cela de les lire, de les interpréter dans un langage faisant consensus - car il est avant tout nécessaire ici de s'entendre sur une langue commune -, ces informations ne valent tout simplement rien, les efforts investis pour garder trace du passé sont anéantis, la mémorisation des observations reste absolument sans valeur. Mieux : cette mémorisation est en elle-même fictive. L'information est certes présente de manière potentielle, mais l'improbabilité des démarches qui permettraient son actualisation est si grande que cette information pourrait en vérité aussi bien ne pas exister. Qui plus est, la plupart des connaissances en médecine étant soumises à un rythme de développement rapide par rapport à d'autres domaines scientifiques (pensons, par exemple, à la physique ou l'astronomie $)^{5}$, les observations en question sont rapidement périssables, et les délais dont on dispose pour les exploiter sont courts. Elles seront rapidement obsolètes, et ne vaudront plus que comme trace de savoirs antérieurs, non comme support pour prendre des décisions dans le présent ou orienter des démarches dans l'avenir.

Pour une large part, l'usage que la médecine cherche actuellement à faire de l'intelligence artificielle (IA) est clairement destiné à surmonter cette désorientation. À ce titre, il ne vise pas seulement à délester le personnel médical de tâches cognitives ingrates, possiblement fastidieuses, ou encore à en augmenter le rendement, mais il a également pour ambition de permettre des opérations cognitives que l'être humain, en raison de sa finitude ${ }^{6}$, $n^{\prime}$ est pas capable de prendre en charge.

4. C'est là ce que B. Bachimont appelle la désorientation. Cf. Bachimont (2004, p. 105-108). 5. Notamment car les connaissances médicales qui prévalent à une époque donnée dépendent fortement des modes de vie des individus (de l'alimentation aux formes du travail), modes de vie soumis à un rythme d'évolution rapide. Pour le dire de manière ramassée, les maladies dont souffrent nos parents ne sont pas les mêmes que celles qui nous affectent.

6. La notion de finitude réfère ici au caractère limité des ressources dont dispose l'être humain pour réaliser ses différentes opérations cognitives. Celles-ci sont principalement limitées du point de vue 1 ) de leur capacité de traitement et de stockage ;2) de leur vitesse. $1^{\circ}$ Seule une quantité limitée d'informations peut être traitée de manière simultanée ou stockée en mémoire (l'homme ne dispose pas d'une mémoire infinie), et seuls des calculs de complexité limitée (notamment au sens du nombre de paramètres manipulés) peuvent être réalisés. $2^{\circ}$ Calculer, planifier, décider, penser sont des opérations qui prennent du temps, et 
Passer en revue des corpus comportant plusieurs centaines de milliers de documents est hors de portée d'un individu isolé, et la solution que prétend apporter le travail collectif est loin d'aller de soi, en ce qu'elle suppose d'une part la disponibilité d'une main d'œuvre importante, mais surtout en ce qu'elle nécessite, d'autre part, de disposer de méthodes opératoires pour partager, et donc extérioriser, et par suite synthétiser les informations extraites par chacun. Cette finitude cognitive de l'humain sanctionne particulièrement la fouille de données et l'extraction de connaissances lorsque ces opérations sont soumises à d'importantes contraintes de temps, comme dans le cadre de la pharmacovigilance, où la détection de signaux d'effets indésirables liés aux médicaments (EIM) doit être rapide, sous peine de conséquences parfois fâcheuses. Disposer de SBC capables de fouiller d'immenses bases de cas, le plus souvent faiblement normalisés, parfois rédigés en langage naturel $^{7}$, pour extraire des signaux d'EIM potentiels, est alors de prime importance ${ }^{8}$.

Les ontologies ont assurément un rôle clef à jouer dans ce processus. Elles peuvent d'une part favoriser la normalisation des données, condition sine qua non de leur exploitation par des SBC. Elles offrent, d'autre part, de projeter sur les données un réseau sémantique rendant leur traitement pour l'extraction de connaissances plus performant, par exemple, en permettant le regroupement de données portant sur des conditions médicales analogues mais présentées sous des libellés différents. Dans le cadre de la pharmacovigilance, différents auteurs ont ainsi insisté sur l'importance de disposer d'un vocabulaire suffisamment spécifique pour coder avec précision les cas d’EIM, mais ont également marqué la nécessité que ces termes soient suffisamment interconnectés pour que les analyses statistiques impliquées par la détection du signal puissent présenter des résultats significatifs ${ }^{9}$. Différents travaux ont par ailleurs montré que le raisonnement sur les définitions formelles de concepts d'ontologies des EIM permettait d'améliorer les performances dans la détection du signal $^{10}$, et facilitait l'accès aux données dans les bases de cas ${ }^{11}$.

Le développement d'ontologies et de systèmes capables de réaliser de telles opérations est cependant loin d'être trivial, et l'ingénierie des connaissances bute sur certains problèmes récurrents, ayant notamment trait à la difficulté de formaliser les connaissances et raisonnements susceptibles de les exploiter, ou à celle de maintenir ces représentations à jour avec des connaissances médicales soumises à un rythme d'évolution extrêmement rapide. Plus radicalement, différents auteurs ont récemment fait le constat sévère que les systèmes d'information pour la santé actuels, qu'ils exploitent ou non des ontologies, étaient un pur et simple échec ${ }^{12}$. Une partie des

une réalisation plus rapide se fait souvent au prix d'une perte de précision (risque d'erreurs accru).

7. Cf. Avillach et al. (2010).

8. Voir par exemple Henegar et al. (2006) ; Nadkarni \& Darer (2010); Avillach et al. (2010).

9. Voir Meyboom et al. (2002); Alecu et al. (2008).

10. Cf. Bousquet et al. (2005); Henegar et al. (2006).

11. Cf. Alecu et al. (2007).

12. Voir Heeks (2006) pour une revue sur la question. 
raisons de cet échec a trait à l'inadéquation de ces systèmes vis-à-vis des habitudes de travail du personnel censé les utiliser, et donc à des dimensions et mécanismes qui sont principalement du ressort des sciences sociales, de l'ergonomie du travail et en un sens de la psychologie. Cependant, si les individus ne parviennent pas à intégrer ces systèmes dans les circuits ordinaires de leurs activités professionnelles (ou ne désirent pas le faire), c'est également que ceux-ci leur proposent, voire leur imposent des manières d'œuvrer qui sont inadaptées à leurs activités de connaissance ${ }^{13}$. Le traitement que les machines font subir aux connaissances médicales pour les manipuler est selon nous au moins en partie responsable de cette situation ${ }^{14}$.

Nous voudrions à ce titre montrer, à partir de l'analyse de différents usages de SBC dans le domaine de la santé, que la plupart des difficultés aujourd'hui rencontrées par l'ingénierie des connaissances médicales sont inhérentes à la nature même de l'IA, en tant que projet de mécanisation de l'activité cognitive. À notre sens, ces difficultés ne sont pas le fait d'un stade encore larvaire de développement de notre expertise dans le domaine de la formalisation des connaissances, encore moins la conséquence d'une limitation actuelle des performances des machines (capacités de stockage ou de traitement de l'information), mais elles sont inhérentes au processus de formalisation qui rend les connaissances computables. Pour étayer cette affirmation, nous commençons par décrire les ontologies et les principes guidant leur conception (section 2); nous présentons ensuite, dans ses grandes lignes, la critique que Dreyfus adresse à l'IA computo-symbolique (section 3); et nous proposons finalement d'analyser certaines des difficultés actuellement rencontrées en ingénierie des connaissances médicales depuis la grille de lecture que nous fournit cette critique (section 4 ).

\section{Ontologies et connaissance}

\subsection{Les ontologies : définition et principes de conception}

Une ontologie, dans l'ingénierie des connaissances contemporaine, correspond à un ensemble hiérarchisé de concepts articulés suivant des relations sémantiques explicites, le plus souvent de subsomption (mais par exemple également de partinomie), et définis par ces relations ${ }^{15}$. Les ontologies peuvent ainsi se représenter sous forme d'arbres ou de treillis conceptuels. Et elles ont le plus souvent pour vocation de formaliser et structurer un ensemble de connaissances, relatives à un domaine spécifié (un univers de référence). Elles organisent, en leur conférant une structure logique, les «catégories et termes permettant de penser un domaine et

13. Cf. Berg \& Toussaint (2003).

14. C'est également l'avis de Bouaud \& Séroussi (2005).

15. Nous ne nous arrêtons pas ici sur les différentes définitions qui ont pu être proposées des ontologies. Les plus souvent retenues sont celles de Gruber (1993), Guarino (1997), Uschold et Gruninger (1996), et de Bachimont (2004). Voir Charlet (2002) pour revue. 
d'exprimer les connaissances que l'on a sur lui $»^{16}$. Elles visent en ce sens à réaliser la grammaire d'un domaine de connaissances existant.

La signification des concepts qui composent une ontologie est généralement considérée comme correspondant à ce qu'on appelle l'extension de ces concepts, c'est-à-dire l'ensemble des individus qui appartiennent à la classe nommée par le concept. La seule manière de définir formellement des concepts ainsi compris est cependant de spécifier leur intension: soit les propriétés que doivent posséder les individus, ou plus généralement les conditions qu'ils doivent remplir pour appartenir à l'ensemble indiqué par le concept - en somme leurs conditions d'éligibilité. Dans les représentations ontologiques, la spécification de l'intension est en partie prise en charge par le positionnement du concept dans le réseau sémantique. S'il s'agit d'un arbre de subsomption, tel concept sera défini comme possédant les attributs que possède son concept «père » (soit le concept qui le subsume). Il s'agit de la logique de l'héritage: si le concept de rein est défini comme ayant un rapport de subsomption avec le concept d'organe - le rein est un organe (relation bien connue sous son appellation anglophone : is a) -, le concept de rein hérite les attributs qui définissent le concept d'organe (par exemple le fait d'être localisé à un endroit donné de l'organisme et de participer à une certaine fonction). Plus généralement, l'intension du concept sera spécifiée par l'ensemble des relations sémantiques (verticales/hiérarchiques aussi bien qu'horizontales/associatives) qui l'articulent aux autres concepts du réseau. Ainsi, les ontologies sont bien plus des réseaux articulant des concepts (ce que les linguistes et psychologues appellent depuis les années 60 des réseaux sémantiques ${ }^{17}$ ), que des systèmes de définitions des concepts. C'est la position qu'occupe le concept dans le réseau sémantique, donc les relations qu'il entretient avec les autres concepts, qui en spécifient la signification.

$\mathrm{Au}$ sens strict, les ontologies n'ont en aucune façon pour fonction de stocker des connaissances. C'est le rôle des bases de données. Leur fonction est uniquement de permettre de décrire des connaissances, en respectant certaines règles de cohérence, le plus souvent empruntées à la logique. Les ontologies mettent à disposition des systèmes conceptuels permettant l'expression des connaissances dans un format autorisant des opérations purement formelles sur celles-ci (soit des opérations ne nécessitant aucune composante interprétative : des calculs), et donc leur réalisation automatique par des machines. Elles décrivent à ce titre les relations formelles entre concepts, non pas les relations empiriques entre les objets auxquels réfèrent les concepts. C'est de cette manière, et de cette manière uniquement, qu'il faut entendre leur fonction de représentation des connaissances.

16. Bachimont (2004, p. 144).

17. Ces réseaux étaient à l'origine destinés à modéliser la manière dont les connaissances de l'individu sont organisées dans sa mémoire, et dont se réalise l'accès à ces connaissances, aussi bien dans l'activité de catégorisation perceptive que dans le raisonnement. Voir par exemple Rosch (1977), Lindsay \& Norman (1980), ou Rastier (2004). 


\subsection{Formalisation des connaissances et computabilité de la signification}

Nous l'avons vu précédemment, les ontologies sont des systèmes de concepts spécifiés par des définitions formelles. À quoi exactement se réfère cette épithète? Que signifie définir formellement un concept ?

Pour le dire de manière synthétique, le formel caractérise ce dont la signification est indépendante de tout contexte, ou plutôt ce qui vaut invariablement quel que soit le contexte (car après tout, il n'y a jamais de signification qu'en contexte). Considéré d'une perspective purement formelle, le concept de carcinome hépatique a toujours la même signification, quel que soit le contexte. Son intension, et donc son extension, ne changent pas selon qu'il est employé dans un service de cancérologie, d'urgences, ou un cours de pathologie générale tumorale.

Un langage formel correspond ainsi à un langage où la signification des formules est intégralement spécifiée par leur forme (soit la syntaxe des énoncés), sans qu'il soit nécessaire de recourir à une étape d'interprétation nécessitant les performances d'un esprit interprète ${ }^{18}$. La force d'un langage formel est de se passer de l'interprétation. Comme dans tout langage, ses formules, si elles sont agencées selon des règles syntaxiques déterminées, signifient quelque chose, mais pour le signifier elles n'ont pas besoin d'un apport herméneutique supplémentaire. Il n'y a pas à prendre en considération le contexte de l'énonciation (par exemple à imaginer les raisons du locuteur qui émet le jugement), ou le référent des symboles. Le caractère contextuel de l'interprétation (la signification est déterminée par le contexte de production de l'énoncé : la signification d'un même énoncé peut donc varier dès lors que le contexte change) et son caractère référentiel (elle prend en considération les référents des termes et non pas seulement leur composition syntaxique), font par principe défaut à l'ordre formel ${ }^{19}$. Définir une proposition ou un concept en langage formel signifie dans cette mesure les définir de manière à les rendre manipulables dans le respect de règles intégralement formulées a priori (par exemple les règles d'inférence de la logique traditionnelle) sans que soit pour cela nécessaire de recourir à aucune interprétation ou composante intuitive. La manipulation peut être réalisée «à l'aveugle», l'inférence peut se faire de manière intégralement mécanique.

Si une compréhension claire de la nature du formel est indispensable pour évaluer ce que l'IA peut apporter à la médecine, et donc également ce qu'elle ne saurait lui apporter, c'est avant tout parce que les machines numériques ne sont capables de manipuler, par définition, que les couches formelles de la signification,

18. Cf. Bachimont (2004, p. 123).

19. Comme l'explique Bachimont, les énoncés exprimés en langage formel «sont non contextuels : si, en effet, l'interprétation dépend du contexte, la forme de l'énoncé ne suffira pas à déterminer sa signification. Le fait que la sémantique soit formelle implique que l'interprétation soit la même quel que soit le contexte, et n'est déterminée que par la forme seule. » (Bachimont, 2004, p. 123). 
soit des «connaissances » réduites à leur seule forme syntaxique. Les machines étant par principe incapables d'interpréter, elles ne peuvent en effet « raisonner » que sur des contenus dont les propriétés sémantiques auront été préalablement formalisées. Dans un algorithme, on ne peut inscrire une instruction que la machine devrait « interpréter» en lui attribuant un sens (par exemple, en lui associant une action à

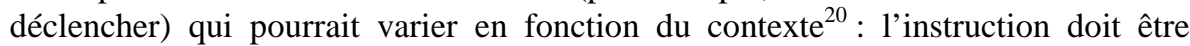
absolument univoque, il ne peut y avoir aucun jeu entre la formule et son sens. L'évacuation de l'interprétation par la réduction au formel (c'est-à-dire à la syntaxe) conditionne la computabilité des contenus.

\section{Dreyfus et les limites de l'IA computo-symbolique}

Les machines ne pouvant manipuler que des connaissances préalablement exprimées en langage formel, il importe de déterminer ce qui, du savoir médical, est réductible à des compositions syntaxiques, pour évaluer ce que l'IA est en mesure d'apporter à la médecine, et pour sanctionner en retour les prétentions à demander aux machines ce que, en raison de leur nature, elles ne peuvent faire. À notre sens, des éléments de réponse à cette question peuvent être trouvés dans certaines des critiques que le philosophe H. L. Dreyfus a adressées à l'IA, des années 1960 à aujourd'hui $^{21}$. Bien que ces critiques aient une portée plus générale que le seul domaine de l'IA appliquée aux connaissances médicales, nous allons voir que la plupart des problèmes mis en évidence par Dreyfus se retrouvent, sous une forme parfois étonnamment proche, dans l'ingénierie des connaissances pour la santé.

Le nerf de la critique ${ }^{22}$ que Dreyfus adresse à l'IA dite cognitiviste ou computosymbolique - ironiquement baptiséee Good Old-Fashioned Artificial Intelligence (GOFAI) par J. Haugeland (1985) - porte sur le caractère non formalisable et non propositionnel des connaissances, ou plus généralement des compétences sur lesquelles repose le comportement intelligent humain, qu'il s'agisse d'un comportement de résolution de problème dans un cadre à première vue hyperformalisé tel que le jeu d'échecs, ou d'un comportement d'adaptation aux

20. À moins bien entendu d'avoir au préalable formalisé les éléments dudit contexte que l'interprétation est susceptible d'exploiter. Nous verrons toutefois plus loin avec Dreyfus les problèmes que soulève une telle entreprise.

21. Dreyfus est aujourd'hui considéré comme un des auteurs de référence en philosophie et épistémologie de l'IA. Il est l'un des premiers critiques de l'IA cognitiviste (il publie ses premiers travaux dans les années 1960, son ouvrage majeur, What computers can't do, est publié en 1972), et également l'un des plus tenaces (il poursuit sa critique encore aujourd'hui, comme en témoignent les travaux qu'il publie régulièrement sur la question). C'est également l'un des mieux informés et des plus conséquents au plan argumentaire. La plupart des limites de l'IA cognitiviste qu'il pointe dans ses premiers ouvrages, se sont, de l'avis de nombreux chercheurs en IA, révélés parfaitement justifiées par la suite (voir par exemple Crevier, 1997). 22. Il faut avant tout entendre ce terme en un sens kantien : en éclairant les présupposés épistémologiques de l'IA, Dreyfus en précise les limites. 
situations de la vie quotidienne, dont la performance a lieu dans un cadre a fortiori moins formalisé. Aussi, cette critique ne porte-t-elle pas sur l'IA en tant que telle, mais de manière plus radicale sur le modèle de l'esprit comme machine symbolique, sur lequel se construit l'IA. Le postulat à la base de l'IA cognitiviste est en effet que les processus cognitifs qui sous-tendent le comportement intelligent consistent pour l'essentiel en des manipulations de représentations symboliques (en particulier des propositions exprimant des croyances : à propos de l'état présent de la réalité, aussi bien que de son état à venir - anticipations) obéissant à des règles formelles. De sorte que si l'on parvient à implémenter dans une machine des algorithmes et systèmes de traitement de l'information, lui offrant de développer des propositions symboliques représentatives de l'état de son environnement, ainsi que les règles de manipulation de ces propositions et de déclenchement du comportement adéquates, on pourra créer un comportement intelligent artificiel de même nature que celui à l'œuvre chez les êtres vivants, humains compris. C'est là l'affirmation essentielle promue par Newell \& Simon (1976) dans leur fameuse Physical Symbol System Hypothesis. En reprenant les affirmations paradigmatiques du cognitivisme, l'IA ne se contente pas d'affirmer qu'il est possible de créer des machines intelligentes, elle postule également - et c'est en vérité de cette manière qu'elle légitime l'affirmation précédente - que l'intelligence possède dans son principe même un caractère machinique.

Pour Dreyfus, cette conception se méprend foncièrement sur la nature de l'intelligence humaine et les «mécanismes » qui la sous-tendent. Le comportement intelligent repose bien plus sur des savoir-faire (know-how) que sur des connaissances propositionnelles, et ces savoir-faire sont fondamentalement non formalisables : les exprimer, comme cherchent à le faire les concepteurs de systèmes experts, sous la forme de listes ou d'arbres de règles, destinés à déterminer une décision adéquate pour toute situation rencontrée, c'est perdre ce qui fait justement l'intelligence du comportement. Le comportement intelligent n'est tout simplement pas un comportement réglé. Et il n'est pas seulement affaire de manipulation logique de symboles, mais il implique l'incarnation (la possession d'un corps et la capacité à agir par le corps sur le monde), l'acculturation (une familiarité avec les normes et habitus culturels), la capacité d'imagination, d'empathie et l'aptitude émotionnelle autant de compétences faisant défaut aux machines symboliques. Ainsi Dreyfus peutil déclarer : "Intelligence requires understanding, and understanding requires giving a computer the background of common sense that adult human beings have by virtue of having bodies, interacting skillfully with the material world, and being trained in a culture." 23

Cette critique, que Dreyfus a notamment explicitée à propos de ce qu'on appelle le problème de la connaissance de sens commun (commonsense-knowledge problem), ne porte pas tant sur la difficulté qu'il y a à représenter cette « connaissance », que sur la nécessité à laquelle se trouve acculé le concepteur de

23. Dreyfus (1992b, p. 3). 
systèmes artificiels intelligents de convertir en connaissances des compétences qui, dans le comportement intelligent, n'en ont justement pas la nature ${ }^{24}$.

Pour appuyer ce propos, Dreyfus mentionne notamment le rapport inverse entre la quantité de connaissances acquises («l'expérience») et la rapidité de réaction à une situation chez l'humain et la machine computo-symbolique. Alors que la machine met d'autant plus de temps à traiter une information et s'adapter à une situation que ses bases de connaissances sont importantes, l'humain va d'autant plus vite et a d'autant plus de facilité qu'il a d'expérience ${ }^{25}$. Pour Dreyfus, cela s'explique par le caractère global et contextuel de la sensibilité de l'être humain aux éléments pertinents d'une situation, contre le procédé analytique, acontextuel et sériel qui caractérise les programmes fonctionnant à base de faits et de règles ${ }^{26}$. Notre capacité à saisir les éléments pertinents d'une situation (et donc à faire le tri, à ne pas prêter attention à ce qui est sans intérêt) est en effet holistique : nous n'avons pas à passer en revue de manière sérielle chacun des éléments composant la situation, pour les catégoriser comme pertinents ou au contraire sans intérêt, étant donné la nature du problème dans lequel nous nous trouvons engagés. Nous appréhendons d'emblée la situation comme un champ global présentant une certaine physionomie, sans avoir pour cela eu besoin de l'atomiser au préalable. Une telle aptitude présuppose par ailleurs pour Dreyfus l'implication concrète de l'agent dans l'activité, elle présuppose que l'activité présente un certain enjeu pour cet agent - qui doit donc disposer d'une forme d'incarnation, de capacité émotionnelle, et plus radicalement de capacité à se sentir concerné par la situation ${ }^{27}$. Alors que les représentations symboliques, à l'inverse, sont toujours atomistiques, acontextuelles et détachées d'une telle activité $e^{28}$. Leur prétention à valoir quelle que soit la situation considérée leur confère un caractère désitué. Ainsi, alors que l'être humain qui est familier d'une situation ne va d'emblée prêter attention qu'à ce qui compte (ne retenir que l'essentiel) et ignorer une foule de détails sans importance, la machine est obligée de passer en revue une par une toutes les informations dont elle dispose pour déterminer «en connaissance de cause", si l'on peut dire, ce qu'elle peut se permettre d'ignorer. Le problème est ainsi que la machine ne peut ignorer certains éléments de la situation qu'après avoir explicitement décidé de les ignorer, donc justement après les avoir pris en considération. La force de l'être humain est qu'il n'a pour sa part

24. "The problem precisely was that this know-how, along with all the interests, feelings, motivations, and bodily capacities that go to make a human being, would have had to be conveyed to the computer as knowledge - as a huge and complex belief system - and making our inarticulate, preconceptual background understanding of what it is like to be a human being explicit in a symbolic representation seemed to me a hopeless task." (Dreyfus, 1992b, p. xi-xii).

25. Cf. Dreyfus (1992b, p. xxi-xxii).

26. Cf. Dreyfus (1992b, p. xxix).

27. "As I taught I wondered more and more how computers, which have no bodies, no childhood, and no cultural practices but are disembodied, fully formed, nonsocial, purely analytic engines, could be intelligent at all." (Dreyfus \& Dreyfus, 1986, p. 5)

28. Cf. Dreyfus (1992b, p. xi). 
pas à considérer d'abord les éléments qu'il va ignorer. Sa vision est d'emblée construite comme ne tenant pas compte de ce qui ne compte pas ${ }^{29}$.

La critique que Dreyfus adresse à l'IA est en vérité enchâssée dans une critique plus large, qui porte sur la conception de la connaissance que promeut la philosophie rationaliste occidentale depuis l'Antiquité. Pour Dreyfus, l'IA cognitiviste n'est au fond qu'un avatar moderne de la théorie rationaliste de la connaissance, qui affirme que «pour tout domaine qui peut être maîtrisé, il existe un ensemble de traits et de règles qui expliquent cette maîtrise $»^{30}$. Quand elle élabore des systèmes experts, l'IA part ainsi du principe que les connaissances de l'expert consistent en des heuristiques ou ensemble de règles (qui peuvent se représenter sous la forme d'instructions : si condition $\mathrm{C}$ alors action $\mathrm{A}$ ) qui possédaient un caractère explicite lors de leur phase de mise en place, mais sont aujourd'hui passées dans un inconscient cognitif permettant leur mise en œuvre silencieuse et automatique ${ }^{31}$. Et en effet, pour le cognitivisme, l'expert ne se rend plus compte des règles qu'il applique et qui sont pourtant la raison dernière de son expertise, il ne se rend plus même compte que c'est en appliquant des règles qu'il agit. Si on l'interroge, il parle de savoir-faire, d'intuition, de connaissance pratique, d'habitus, mais c'est uniquement que les règles formelles qu'il applique sont trop enfouies dans son système cognitif pour pouvoir émerger à la surface de son champ de conscience de manière à être verbalisées. Il les applique en somme à son insu.

Pour Dreyfus, cette conception rationaliste est fourvoyante. Elle travestit la nature aussi bien que le fonctionnement effectif de la connaissance. On peut s'en rendre compte par un examen phénoménologique minutieux de son processus de mise en place. Si bien souvent les premières phases de développement d'un savoirfaire reposent effectivement sur la mise en application explicite de règles transmises par des pairs, le développement progressif d'une expertise véritable consiste justement dans la mise en place d'une capacité d'adaptation ne reposant plus sur l'application de règles, fussent-elles internalisées et inconscientisées, mais sur une sensibilité holistique aux traits pertinents des situations ${ }^{32}$. «L'expert n'applique pas de règles », «il se borne à [...] discriminer des milliers de cas particuliers $»^{33}$. On ne saurait donc, en l'exhortant à expliciter les prétendues règles sur lesquelles il se base pour agir, que parvenir à des reconstructions purement artificielles. Mieux : «si l'on demande des règles à un expert, on le force en fait à régresser au niveau d'un débutant et à énoncer les règles dont il se souvient encore, mais qu'il n'utilise plus $»^{34}$. Ainsi, pour Dreyfus, si l'on veut comprendre «ce qu'est un savoir-faire et en quoi consiste l'expertise acquise par l'expert», on doit «abandonner l'idée

29. Cf. Dreyfus (1992b, p. xxviii-xxix).

30. Dreyfus (1992a, p. 372).

31. Cf. Dreyfus (1992a, p. 354).

32. Comme le dit Dreyfus: "There is no reason to think that the rules that play a role in the acquisition of a skill play a role in its later application." (Dreyfus, 1992b, p. xiii).

33. Dreyfus (1992a, p. 366-367).

34. Dreyfus (1992a, p. 367). 
traditionnelle selon laquelle un débutant commence par des cas particuliers pour ensuite, à mesure qu'il progresse, en dégager par abstraction des règles de plus en plus sophistiquées ${ }^{35}$. Car c'est en effet précisément le contraire qui se passe : «l'acquisition d'un savoir-faire [consiste] à passer de règles abstraites à des cas particuliers $»^{36}$.

Ainsi, un expert aux échecs n'a pas besoin de planifier ses coups en les subordonnant à une stratégie explicitement formulée et décidée à l'avance ${ }^{37}$. Cette stratégie est comme encapsulée dans sa manière de percevoir, d'organiser et de donner sens aux situations auxquelles il se voit confronté, il est capable, comme l'explique Dreyfus dans la suite d'Herbert Simon, « de saisir presque immédiatement une position et le coup qu'elle lui commande de faire $»^{38}$. Le joueur expert ne joue donc pas sans stratégie, mais la stratégie qui commande ses coups ne lui est pas présente à l'esprit sous forme d'une métarègle dictant les règles particulières à appliquer pour chaque configuration de l'échiquier. Et elle ne peut comme telle se voir explicitée qu' après coup, à travers une reconstruction a posteriori $^{39}$.

Étant donné la nature non formalisable du savoir-faire humain, il est pour Dreyfus illusoire de vouloir construire des systèmes computo-symboliques à base d'instructions pour recréer artificiellement ce savoir-faire. Le développement d'une base de connaissances capable de répertorier tous les éléments de situation sur lesquels l'expert se base prétendument pour agir est une tâche infinie et qui reste aveugle au rôle déterminant de ce que Dreyfus appelle l'arrière-plan, aussi bien qu'à la fonction que remplissent l'imagination et l'incarnation, dans cette expertise. Il est donc tout bonnement préférable de renoncer à l'ambition de robotiser les caractères du comportement humain qui sont par définition contraires au fonctionnement formel des machines symboliques. Ainsi pour Dreyfus du caractère de flexibilité inhérent au comportement humain, soit sa capacité à s'adapter à l'imprévu, l'aléa, l'inédit. Plutôt que de chercher à créer des machines au comportement flexible (tâche selon Dreyfus impossible du fait du caractère foncièrement accidentel de la réalité où nous évoluons), il est préférable de normaliser les situations auxquelles va se trouver confrontée la machine ${ }^{40}$.

35. Dreyfus (1992a, p. 356).

36. Dreyfus (1992a, p. 356).

37. Cf. Dreyfus (1992a, p. 364).

38. Dreyfus (1992a, p. 365).

39. Cf. Dreyfus (1992b, p. xxviii).

40. "No program can deal flexibility with components of arbitrary shapes or with unanticipated failures that a human being would easily detect. It seems wiser, then, to abandon the goal of human flexibility and seek engineering solutions consistent with the limited capacities of present-generation robots. Better methods of standardization can obviate the need of human flexibility, and they have the advantage of working." (Dreyfus \& Dreyfus, 1986, p. 185). 
Ce verdict de Dreyfus n'est pourtant pas une condamnation de l'IA, mais uniquement la condamnation du projet cognitiviste de réduction de l'intelligence humaine à un système computo-symbolique. Ce qui ne veut pas dire non plus que l'IA computo-symbolique soit en tant que telle un pur et simple échec, mais uniquement que des machines opérant sur la base de ces principes ne vont pouvoir être efficaces que dans des domaines d'activité limités. Pour Dreyfus, on ne peut ainsi développer de systèmes computo-symboliques performants que si le domaine de connaissances sur lequel ils opèrent est d'avance circonscrit et normalisé - en bref, des systèmes dont le domaine peut être traité comme un jeu, soit une activité pour laquelle il existe des règles explicites, un ensemble fermé de situations et de possibilités d'action, et où les critères de pertinence des décisions peuvent être déterminés de manière univoque (c'est-à-dire sans avoir à prendre en considération le contexte dans lequel le jeu se déroule $)^{41}$. Autrement dit, est machinisable tout domaine d'expertise où ce qui est pertinent est décidable et formalisable par avance. Mais ne sont en revanche en aucune manière machinisables les domaines d'expertise où la détermination de ce qui est pertinent est précisément ce qui pose problème ${ }^{42}$.

\section{Le problème de la formalisation de la connaissance en ingénierie des connaissances médicales}

Les démarches actuellement menées en ingénierie des connaissances médicales, en particulier dans le domaine de la conception de SBC exploitant des ontologies, échappent-elles aux critiques de Dreyfus? Se conforment-elles à ses recommandations? Ou bien l'informatique médicale actuelle s'est-elle, comme l'IA cognitiviste, engagée dans une voie que Dreyfus considérerait comme sans issue ? Des éléments de réponse à ces questions peuvent être apportés sur plusieurs fronts.

\subsection{La formalisation du langage naturel et le problème du contexte}

Tout d'abord, le constat aujourd'hui répandu parmi les concepteurs d'ontologies et SBC qu'il est nécessaire de développer des systèmes focalisés, spécifiques à un domaine de connaissance hautement spécialisé et des tâches bien circonscrites, et non pas généraux ${ }^{43}$, va manifestement dans le sens de Dreyfus ${ }^{44}$. Se cantonner à un

41. "As long as the domain in question can be treated as a game, i.e., as long as what is relevant is fixed, and the possibly relevant factors can be determined in terms of context-free primitives, then computers can do well in the domain. [...] In such special-purposed programs the form of knowledge representation can be limited to situation $\rightarrow$ action rules in which the situation is defined in terms of a few parameters and indicates the conditions under which a specific heuristic rule is relevant." (Dreyfus, 1992b, p. 27-28).

42. Cf. Dreyfus (1992b, p. 33).

43. Voir Charlet (2002, chapitre 2). 
domaine spécifique que l'on va être en mesure de circonscrire (soit ce qu'on appelle depuis les années 1970 en IA un «micromonde»), c'est réduire l'incertitude des situations que la machine va avoir à traiter, aplanir les singularités - et c'est en ce sens se donner les moyens de formaliser les critères de pertinence que va pouvoir exploiter le système pour traiter les données. De même, la difficulté, largement reconnue, de développer des systèmes capables de prendre en charge l'usage souple du langage fait par l'humain (par exemple le problème de la polysémie ${ }^{45}$ ) et la dépendance de la signification relativement au contexte d'énonciation peut être vue comme une expression du caractère informalisable de ce que Dreyfus appelle l'arrière-plan. À ce titre, les tentatives de développer des SBC capables d'«interpréter » la signification d'énoncés rédigés en langage naturel, en prenant notamment en considération des éléments du «contexte», tombent clairement sous le coup des critiques qu'il adresse à l'IA computo-symbolique. Dès lors qu'on a affaire à des énoncés en langage naturel ou pseudo-naturel, expliciter et formaliser les éléments du contexte d'énonciation, ou relevant de ce que Dreyfus appelle l'arrière-plan, afin de conférer une signification univoque aux énoncés analysés, est, pour reprendre une expression de E. Husserl, une tâche infinie ${ }^{46}$. Le contexte ayant une structure horizontale (il est infiniment déployable dès lors qu'on s'engage dans son explicitation) et réticulaire (toute connaissance constituant le contexte d'une proposition et permettant sa compréhension tient elle-même sa signification d'un nouveau contexte, et ainsi de suite), c'est par principe qu'il ne peut être explicité de manière exhaustive. Toujours interviendront des énoncés nouveaux dont la compréhension nécessitera la prise en compte d'éléments du contexte n'ayant jusqu'alors pas été explicitement identifiés et listés. L'interprétation d'énoncés en langage naturel repose par ailleurs sur une capacité d'imagination, une capacité de mise en situation virtuelle, dont il parait illusoire de vouloir doter la machine, notamment car cette capacité présuppose bien souvent un ensemble d'aptitudes comportementales rendues possibles par le caractère incarné du sujet et sa familiarité avec les habitus culturels de sa communaute ${ }^{47}$.

44. En vérité, il rejoint presque mot pour mot le constat que faisait Dreyfus en 1972 dans What computers can't do, ou celui du mathématicien James Lighthill, interrogé l'année suivante par le Science Research Council anglais au sujet de l'IA : "To sum up [...] work [...] during the past twenty-five years is to some extent encouraging about programs written to perform in highly specialized problem domains, when the programming takes very full account of the results of human experience and human intelligence within the relevant domain, but is wholly discouraging about general-purpose programs seeking to mimic the problem-solving aspects of human Central Nervous System activity over a rather wide field. Such a general-purpose program, the covered long-term goal of AI activity, seems as remote as ever." (James Lighthill, cité dans Dreyfus \& Dreyfus, 1986, p. xii).

45. Voir par exemple l'étude de Pisanelli et al. (2004).

46. Cf. Dreyfus (1992b, p. 57).

47. Cf. Dreyfus (1992b, p. xix-xx). Dreyfus explique ainsi, à propos du programme développé par E. Charniak pour analyser des histoires pour enfants: "So the data base would have to contain an account of all possible exceptions to augment the text - if it even makes sense to 
Ce problème renvoie notamment aux difficultés que rencontre aujourd'hui l'ingénierie des connaissances lorsqu'elle cherche à développer des outils de génération ou de complétion automatique d'ontologies, capables de les enrichir et de les mettre à jour sans avoir à recourir à des experts du domaine considéré. Aucun système efficace de mise à jour, qui pourrait se passer de l'intervention humaine, ou au moins voir cette intervention réduite à un simple processus de validation, n'a à notre connaissance été à ce jour développé ${ }^{48}$. Comme l'enrichissement et la mise à jour automatiques des ontologies ne peuvent $a$ fortiori se réaliser que sur la base de documents, rédigés le plus souvent en langage naturel, leur possibilité dépend de la disponibilité d'outils capables d'en gérer les spécificités afin d'extraire les données pertinentes et de les exprimer, sans assistance humaine, dans un langage formel. On retombe donc sur le problème précédent.

Le problème soulevé par Dreyfus de l'impossibilité d'être exhaustif dans la spécification des connaissances dont devrait disposer une machine pour interpréter des énoncés en langage naturel (soit les connaissances d'arrière-plan) se retrouve également dans l'exigence d'exhaustivité des intitulés textuels des concepts qu'il peut être nécessaire d'atteindre pour qu'un système d'indexation de documents médicaux exploitant une ontologie fonctionne avec un niveau de réussite satisfaisant. Un SBC permettant l'indexation automatique de dossiers patients ${ }^{49}$ pourra permettre de décider plus rapidement d'un diagnostic et des conduites à tenir pour la prise en charge du patient. Mais pour être opérationnel en situation, il doit être capable de s'accommoder des éventuelles «imperfections » du langage dans lequel se trouvent rédigés les documents. Un tel système fonctionne globalement comme suit : chaque chaîne de caractères est prise en entrée dans l'algorithme, qui va chercher à établir une correspondance (le cas échéant, via des méthodes de traitement automatique des langues (TAL)) avec le système de concepts de l'ontologie. Si la chaîne de caractères a pu être appariée avec un concept, le concept servira à indexer le document (phase dite d'interprétation sémantique) ${ }^{50}$. Or, les dossiers médicaux étant le plus souvent rédigés en langage naturel (ou en tout cas dans le langage seminormalisé propre à l'activité médicale concrète), pour que l'interprétation sémantique atteigne un niveau satisfaisant, il est bien souvent nécessaire de disposer de l'ensemble des variations lexicales (synonymes, formes abrégées, etc.) que peut

think of this as a definite list. Worse, even if one listed all the exceptional cases where one would be glad to possess more than one specimen of a certain object, there are situations which allow an exception to this exception [...] The computer programmer writing a story understander must try to list all possibly relevant information, and once that information contains appeals to the normal or typical there is no way to avoid an infinite regress of qualifications for applying that knowledge to a specific situation." (Dreyfus, 1992b, p. 59).

48. Cf. Hitzler et al. (2005).

49. Comme c'est le cas dans le projet LERUDI (LEcture Rapide en Urgence du Dossier Informatisé du patient), qui vise à développer une ontologie (OntolUrgences) qui, intégrée à un SBC, permettra le codage et l'indexation de dossiers patients pour la prise en charge dans des services d'urgences. Voir Charlet et al. (2009).

50. Cf. Mazuel \& Sabouret (2007). 
présenter la forme textuelle du concept. Si une forme rencontrée dans le dossier n'a pas été spécifiée dans l'ontologie, le dossier ne sera pas indexé avec le concept correspondant. Le terme n'apparaîtra pas lorsque l'utilisateur parcourra l'interface. Il devra alors s'accommoder d'une information incomplète, voire erronée ${ }^{51}$.

\subsection{Le problème de la normalisation des données}

La démarche actuelle consistant à normaliser les données que les SBC vont traiter (ainsi des bases de cas utilisées pour la détection du signal en pharmacovigilance ${ }^{52}$ ) est une réponse possible au problème précédent ${ }^{53}$. En vérité, elle consiste très exactement à suivre le conseil de Dreyfus d'améliorer la standardisation plutôt que de chercher à installer de la flexibilité dans les programmes des machines ${ }^{54}$. Plutôt que de concevoir des machines suffisamment tolérantes pour se comporter adéquatement dans un monde humain foncièrement accidentel, on normalise le monde où va opérer la machine. On le rend régulier.

Cette démarche a cependant ses limites et sa mise en œuvre ne va pas sans poser des problèmes qui sont peut-être insolubles dès lors qu'on prend en considération la réalité du facteur humain. Il est en effet loin d'être acquis qu'une telle normalisation des documents soit réalisable, que ce soit au niveau de la saisie ou à travers un posttraitement. Les démarches pour la normalisation des documents lors de la saisie rencontrent ainsi des résistances parfois très fortes chez les acteurs ${ }^{55}$. Amener le personnel de santé à faire usage d'un vocabulaire contrôlé - par exemple le système terminologique de la Snomed-CT (Systematized Nomenclature of Medicine-Clinical Terms) - paraît à de nombreux égards irréalisable. D'une part, parce que la rédaction en langage contrôlé prend beaucoup plus de temps qu'en langage naturel (en tout cas ce langage à demi-normalisé propre à la médecine concrète : qui reste normalisé par les pratiques, non par des systèmes de normalisation qui leur sont extrinsèques). D'autre part, car le médecin ne dispose de toute façon généralement pas de la mémoire suffisante (ou cette fois encore du temps qui serait nécessaire à son développement) pour s'approprier le vocabulaire contrôlé en question. Il ne s'agit

51. Mohammed \& Sahroni (2010) ont avancé l'idée qu'une des principales raisons du rejet des systèmes d'information médicaux avait trait à la qualité de l'information délivrée par ces systèmes. On le comprend bien dans un cadre tel que la prise en charge d'un patient dans un service d'urgences : si le système fournit une information erronée ou incomplète sur le passé médical du patient, les conséquences peuvent s'avérer dramatiques.

52. Cf. Avillach et al. (2010).

53. Cf. Cimino (2007).

54. Voir supra le passage cité de Dreyfus \& Dreyfus (1986), p. 185. De même, Dreyfus \& Dreyfus expliquent, cette fois à propos de la machinisation du travail industriel : "Only if the production process is rationalysed to the point where irregularities are fully defined and contained, thus eliminating the need for the invisible interventions that keep today's factories going, can total automation hope to work." (Dreyfus \& Dreyfus, 1986, p. 186).

55. Cf. Bousquet et al. (2008). 
bien entendu pas ici d'en acquérir une connaissance exhaustive : c'est là le rôle des systèmes d'information qui sont à disposition des médecins. Mais il faut néanmoins le connaitre suffisamment pour savoir s'orienter dans ces systèmes.

Une illustration concrète de ces difficultés nous est fournie par la manière dont se pose le problème de la normalisation des rapports de cas dans le cadre de la pharmacovigilance. Dans le meilleur des mondes, chaque cas de pharmacovigilance répertorié (association d'un ou plusieurs médicaments et d'un ou plusieurs effets indésirables) le serait de manière suffisamment exhaustive pour éviter l'équivoque, les résultats d'examen seraient indiqués de manière précise, et le patient serait décrit avec autant d'exactitude que le permet l'extraction de données en situation. Les heuristiques qu'il serait alors possible de développer pour extraire des signaux d'EIM des bases de cas disponibles seraient vraisemblablement plus performantes qu'elles ne le sont actuellement. Mais quand on prête attention au témoignage des acteurs de la santé, on voit bien que ce monde est loin d'être à notre portée. En particulier, si les médecins ou le personnel hospitalier devaient, pour chaque patient, normaliser manuellement l'ensemble de ces données, à l'aide de thésaurus par exemple, le coût temporel serait tout bonnement inacceptable. Le mieux que l'on puisse faire est manifestement de créer des organismes tels que les centres régionaux de pharmacovigilance (CRP) en France, qui auront pour fonction de centraliser les rapports de cas transmis par les médecins, de les normaliser et d'évaluer leur pertinence à l'aide de différents indicateurs.

Bien entendu, la mise en place d'organismes chargés de centraliser et de normaliser les documents d'abord rédigés dans des formats retenant encore du langage naturel, ce qui le rend non traitable par les machines, présente également un coût qui peut rapidement devenir important, aussi bien en termes de forces humaines que d'infrastructures. Qui plus est, une normalisation des documents postérieure à leur saisie n'échappe pas au problème de la formalisation de la signification, inhérent à toute tentative de ce type : lorsqu'il fait usage d'un langage pseudo-naturel pour rédiger ses documents, le médecin tire parti de tous les caractères qui rendent si difficilement formalisable les énoncés en langage naturel : en particulier le recours à l'implicite, c'est-à-dire ce fonds de savoir et de savoir-faire dont dispose tout praticien et qui lui permettra de comprendre le rapport lorsqu'il le lira. L'attribution d'une signification aux énoncés présuppose ainsi la disponibilité d'un arrière-plan non explicite leur servant de cadre d'interprétation ${ }^{56}$, et les énoncés peuvent le cas échéant posséder une signification en partie ouverte, qui pourra être précisée ultérieurement. Autrement dit, le médecin n'a pas besoin de tout expliciter, il peut s'accommoder de régions de flou. Pour toutes ces raisons, une normalisation des

56. L'interprétation des énoncés peut également, comme nous l'expliquions plus haut, requérir une capacité à imaginer, par exemple, pour se représenter la situation à laquelle le rapport fait référence et la manière dont nous-mêmes aurions pu agir dans ces circonstances. Un tel recours à l'imagination, pour se mettre virtuellement en contexte, offre bien souvent de lever les ambigüités du langage naturel. Voir Dreyfus (1992b, p. xix-xx). 
documents postérieure à la saisie peut rapidement devenir problématique. Et le cas échéant il pourra être nécessaire de reprendre contact avec le médecin pour obtenir des précisions sur tel ou tel point de son rapport, pour expliciter ce qu'il a laissé dans l'implicite, ou qu'il n'a pas jugé bon (ou pas eu le temps) de noter ${ }^{57}$.

\subsection{Le problème de la formalisation des connaissances}

Un autre point méritant ici d'être abordé concerne plus directement le problème de la formalisation des connaissances. On peut d'emblée remarquer que parce qu'elle reprend purement et simplement la conception cognitiviste de la connaissance et du raisonnement (manipulation de représentations symboliques par application de règles formelles : soit ce qu'on appelle des inférences), l'ingénierie des ontologies contemporaines s'expose aux critiques que cette dernière s'est vu adresser $^{58}$. Certes, son entreprise n'ayant a fortiori pas la prétention à modéliser, encore moins à expliquer le fonctionnement de la connaissance (perspective spécifiquement théorique), mais celle de réaliser des dispositifs capables de remplir certains attendus pratiques, ces critiques ne sauraient la concerner de la même manière. Mais elle ne saurait non plus pour cette seule raison s'y soustraire. Si les attendus pratiques en question sont d'assister le raisonnement humain, de prendre en charge certaines de ses étapes ${ }^{59}$, voire dans les cas les plus ambitieux de s'y substituer, elle s'y trouve également exposée.

Nous l'avons vu, l'expression formelle des propriétés sémantiques des concepts permet de réaliser des raisonnements intégralement automatiques sur les ontologies. L'opération dite de classification repose ainsi sur des inférences ne nécessitant pas d'interprétation sémantique engageant un agent humain. Le «sens » manipulé étant

57. Des études ont ainsi montré que $4 \%$ seulement des EIM étaient en moyenne rapportés par le personnel de santé via les circuits de rapport spontané (cf. Bégaud et al., 2002). Ce chiffre est d'autant plus parlant que le personnel de santé est normalement soumis à l'obligation légale de rapporter ces cas. Une des raisons principales est l'investissement temporel de la démarche: non seulement le médecin doit prendre le temps de rédiger un rapport de pharmacovigilance, mais une fois le rapport envoyé, il est fréquent que le personnel du CRP le contacte pour obtenir des précisions sur le cas présenté dans son rapport (cf. Jamet, 2011). La nécessité de cette demande d'information supplémentaire est bien entendu à relier à la démarche d'explicitation des connaissances inhérente à ce travail de normalisation.

58. Celle de Dreyfus, bien sûr, mais également par exemple celle de J. Searle. Voir Steiner (2005), p. 33, pour un résumé de celle-ci.

59. Dans le cas des procédures de détection du signal en pharmacovigilance, on cherche ainsi à mettre en place des systèmes d'extraction de connaissances qui soient capables de réaliser la première étape de l'opération : à savoir l'identification, sur la base de calculs statistiques, de couples de médicaments et d'effets indésirables potentiellement liés causalement. Un tri est donc déjà effectué par la machine, ainsi qu'une classification (attribution d'un indice de probabilité, par exemple sous forme d'indice de disproportionnalité), qui va orienter le jugement humain ultérieur. Cf. Henegar et al. (2006). 
intégralement réduit à des propriétés syntaxiques, aucune contextualisation ou prise en compte du référent concret du concept n'est requise pour opérer le raisonnement. On peut toutefois se demander si la sémantique des concepts dont l'être humain fait usage dans ses comportements de connaissance effectifs est intégralement ou même partiellement formalisable.

Les théoriciens des systèmes experts y ont beaucoup insisté lorsque le développement de ces systèmes était la première préoccupation de l'ingénierie des connaissances: la principale difficulté rencontrée lors du développement d'un système expert est l'extraction et la formalisation, généralement sous forme de faits et de règles, des connaissances sur laquelle l'expert semble s'appuyer pour agir. Il faut rencontrer les agents humains détenteurs du savoir, trouver moyen de le leur faire expliciter, et ensuite le formaliser de manière à ce qu'il puisse, implémenté sous forme algorithmique, réguler le comportement d'une machine.

Dans le cas de la conception d'ontologies, à première vue il ne s'agit pas d'externaliser un savoir d'abord internalisé dans des agents humains. Généralement, le savoir dont on part se trouve déjà externalisé (en tout cas en bonne partie), puisqu'on le trouve dans des documents, des corpus de textes (par exemple des guides de bonnes pratiques en médecine) ou des systèmes terminologiques comme des thésaurus. Il possède, qui plus est, déjà une forme linguistique, donc syntaxique, ce qui désamorce le problème de devoir formaliser quelque chose qui n'est peut-être justement pas, de par sa nature, formalisable. Une des premières étapes de la conception d'ontologies médicales, quand on adopte une méthodologie ascendante (bottom-up), consiste ainsi le plus souvent à analyser, avec des outils de TAL, le contenu de documents produits en activité par les professionnels de santé, de manière à extraire (en mobilisant cette fois des méthodes statistiques) un ensemble de termes susceptibles d'être parmi les plus structurants pour le domaine de connaissances considéré - soit les termes qui sont spécifiques et essentiels au domaine -, afin de les convertir en concepts ${ }^{60}$. Ces termes sont généralement appelés termes candidats en référence à l'opération d'élection qui, le cas échéant, les sélectionnera pour faire partie de l'ontologie.

Une telle procédure court-circuite en partie les problèmes rencontrés au niveau de l'étape d'extraction de connaissances lors de la conception de systèmes experts : les connaissances et concepts existant déjà dans des documents, ils se trouvent déjà explicités et n'ont pas à l'être une seconde fois. Il n'y a apparemment pas ici à convertir un savoir qui pourrait fort bien n'exister que sous une forme implicite en savoir explicite. Et on ne s'expose donc pas au problème des reconstructions artificielles a posteriori.

Toutefois, c'est une illusion de croire que ce serait totalement le cas. En effet, le concepteur d'ontologies n'est en aucune façon dédouané de devoir formaliser ces concepts extraits sous forme de termes-candidats dans un langage qui permettra leur

60. Cf. Mazuel et Charlet (2009). 
manipulation par la machine - donc de devoir convertir certaines dimensions de la sémantique des termes considérés en propriétés syntaxiques. Le format linguistique sous lequel se trouvent de prime abord disponibles les concepts ne constitue pas encore un format manipulable par la machine. Notamment car, comme nous l'avons vu, il s'agit encore de significations ouvertes, ambigües en un sens, qui ne recevront une détermination univoque de l'agent-interprète que par la prise en compte du contexte. Le concepteur doit donc fermer la signification des concepts, ce qu'il fera en les organisant en réseaux sémantiques, c'est-à-dire en les reliant par des relations formelles, en premier lieu la relation de subsomption (axe sémantique généralitéspécificité).

Or, spécifier les relations formelles qui articulent les concepts d'une ontologie, c'est bien chercher à formaliser des relations qui ne présentent pas d'emblée un caractère explicite ou formel. En effet, ces relations ne se trouvent pas explicitement décrites dans les thésaurus, encore moins dans les documents de travail (par exemple les ordonnances ou rapports de diagnostic). On retombe donc sur le problème de devoir expliciter et formaliser une connaissance qui n'a peut-être pas, dans son mode opératoire, de caractère explicite et formel.

Si l'on part du principe rationaliste que les connaissances que l'on va chercher à représenter dans l'arbre conceptuel possèdent déjà, en tant que connaissances existantes et exploitées par des agents humains engagés dans des situations concrètes, un caractère formel, interroger un médecin pour l'amener à définir tel concept médical, signifiera simplement lui demander d'expliciter une connaissance qui possède déjà un caractère formel dans sa sphère d'application d'origine. Mais nous avons vu qu'une telle position était fort critiquable. Pour Dreyfus, cette conception de la connaissance est tout bonnement illusoire. Développer une expertise ne signifie en aucune façon impliciter des connaissances et règles qui auraient au commencement un caractère explicite. Le recours à des plans, des règles ou des connaissances déclaratives pour gouverner l'action, ne concerne que les premières phases d'acquisition d'un savoir-faire expert. Le novice se guide sur des règles explicites (la «notice», le «mode d'emploi», le «guide de bonnes pratiques ») pour développer un savoir-faire. En revanche, les phases ultérieures de l'acquisition se caractérisent par un total passage dans le domaine, non de l'implicite (car il ne s'agit justement pas de règles explicites ayant été implicitées à l'usage), mais du savoir-faire «rule-free », c'est-à-dire qui ne repose pas sur une capacité à appliquer des règles. Les règles explicites (en bref : la «théorie ») ne constituent pas le fond rationnel du savoir-faire de l'expert, qu'il aurait enfoui dans les couches les plus souterraines de son inconscient cognitif. Elles ne sont qu'une sorte de premier déambulateur qui en facilite la mise en place. 


\subsection{Le problème de la formalisation du raisonnement}

À la question précédente de la formalisation des connaissances se trouve directement liée celle de la formalisation des raisonnements, opérations qui manipulent des connaissances et qui permettent d'en développer de nouvelles. Les formes de «raisonnement » que les machines numériques sont capables de réaliser, à savoir ce qu'on appelle traditionnellement des inférences, permettent-elles de modéliser et reproduire le raisonnement médical ? Et sinon, comment peuvent-elles l'assister?

L'inférence peut se définir comme une opération (qu'on qualifie parfois de mentale, mais le terme semble inapproprié si on attribue également aux machines la capacité d'inférer) qui consiste à tirer de propositions de départ tenues pour valide (les prémisses), et en appliquant des règles exprimées de manière purement formelle (règles de combinaison syntaxique), une proposition d'arrivée valide (la conclusion). Les règles en question étant admises, la validité de la conclusion est ainsi assurée par la validité des prémisses. Prise dans ce sens particulier, l'inférence se réduit à une pure et simple déduction: la conclusion est une pure conséquence formelle des prémisses. Et il n'y a donc pas ici, à proprement parler, production de connaissances nouvelles : la conclusion était virtuellement contenue dans les prémisses.

Il faut toutefois noter qu'on distingue également d'autres formes d'inférences, en particulier l'induction (la généralisation à partir d'une pluralité de cas particuliers) et l'abduction (la détermination d'une hypothèse explicative à partir d'une pluralité de cas particuliers $)^{61}$. La différence de ces deux opérations par rapport à la déduction est que la validité des conclusions n'y est pas assurée de manière nécessaire par la validité des prémisses. Il s'agit uniquement d'hypothèses qui se trouvent favorisées en vertu de leur plausibilité.

Il est important de bien les distinguer, car quoiqu'on en dise, il n'est pas du tout acquis qu'elles soient toutes les trois réalisables par des machines numériques. La déduction étant par définition une opération consistant à dériver de propositions de départ une proposition d'arrivée par application rigide de règles formelles, les machines numériques peuvent assurément opérer des déductions. Il ne s'agit là que de combinatoire, de calcul ${ }^{62}$. Mais sont-elles également capables de réaliser des

61. Induction, déduction et abduction sont notamment les trois types de raisonnement formalisés par C.S. Peirce.

62. Encore que tous ne soient pas d'accord pour attribuer aux machines la capacité même de calculer. Le philosophe Fred Dretske explique ainsi : "Can computer add? [...] The following argument is an attempt to show that whatever it is that computers are doing when we use them to answer our arithmetical questions, it isn't addition. Addition is an operation on numbers. We add 7 and 5 to get 12, and 7, 5, and 12 are numbers. The operations computers perform, however, are not operations on numbers. At best, they are operations on certain physical tokens that stand for, or are interpreted as standing for, numbers. Therefore, computers don't add." (Dretske, 2005, p. 283-284). 
inductions et des abductions ? Tout dépend du type de compétences requis par ces modalités de raisonnement: si une forme de capacité d'imagination ou disons, de simulation, s'y trouve nécessairement impliquée $e^{63}$, ou encore une capacité à choisir parmi différentes propositions en vertu de leur plausibilité, l'affirmation est clairement compromise - à moins, bien entendu, d'appeler par exemple «imagination» l'opération consistant à tester l'une après l'autre des hypothèses préalablement listées et formalisées dans des bases de connaissances, ou de considérer le calcul d'un indice mathématique comme une procédure d'évaluation de la «plausibilité » d'une hypothèse (par exemple d'un diagnostic). Mais dans ce cas, la machine ne réalise par réellement d'induction ou d'abduction, elle se contente de déduire, par application mécanique des règles formelles implémentées dans ses algorithmes, des propositions à partir d'autres propositions ou de données d'entrée ${ }^{64}$.

Ainsi, selon cette conception des formes logiques du raisonnement, les machines ne pourraient par principe réaliser que des déductions, c'est-à-dire inférer des propositions sur un mode analytique, non des inductions ou des abductions, c'est-àdire des propositions synthétiques. Les propositions issues de la procédure de déduction logique étant virtuellement contenues dans les axiomes de départ, la déduction constitue en effet une opération purement analytique: elle ne produit aucune connaissance réellement nouvelle. L'induction et l'abduction sont au contraire des opérations synthétiques, au sens où elles permettent d'enrichir les connaissances disponibles de propositions qui ne sont pas déductibles a priori (c'està-dire indépendamment de toute considération empirique) des prémisses.

Le raisonnement scientifique, raisonnement médical y compris, exploitant manifestement ces trois formes de raisonnement, qui y trouvent une complémentarité, cela signifie que ne pourraient être formalisées et réalisées par une machine que les étapes de celui-ci correspondant à de pures déductions (si tant est qu'il y ait un sens à isoler ces étapes de l'opération globale dont elles participent ${ }^{65}$.

63. Comme le tient par exemple le philosophe et linguiste M. Johnson : "Imagination is a pervasive structuring activity by means of which we achieve coherent, patterned, unified representations. It is indispensable for our ability to make sense of our experience, to find it meaningful. The conclusion ought to be, therefore, that imagination is absolutively central to human rationality, that is, to our rational capacity to find connections, to draw inferences, and to solve problems." (Johnson, 1987, p. 168, cité dans Dreyfus, 1992b, p. xxi).

64. Ainsi, les principales formes de raisonnement utilisées sur les ontologies:1) la classification : réorganisation des rapports de subsomption entre les classes à partir de leurs définitions formelles, et vérification que telle classe est bien contenue dans telle autre par analyse de leurs définitions ; 2) l'évaluation de la consistance : vérification que les critères d'appartenance à une classe ne sont pas contradictoires, sont toutes deux réductibles à des inférences déductives.

65. Voir à ce titre les nombreux travaux que J. Bouaud et B. Séroussi ont consacré à l'informatisation des guides de bonnes pratiques. Insistant sur le caractère décontextualisé des connaissances formalisées dans les systèmes d'aide à la décision traditionnels, ainsi que sur le caractère informalisable du contexte qu'exploite le médecin dans ses raisonnements situés, 
Par ailleurs, une approche formaliste de la connaissance et du raisonnement médical semble laisser échapper ce qui en constitue pourtant, à en croire différents théoriciens, un trait essentiel : à savoir son caractère collectif et distribué. Comme l'ont expliqué Berg \& Toussaint (2003) et d'autres avant eux, les connaissances médicales sont le fruit d'un travail d'élaboration collectif, où la dimension socionormative est prépondérante, et qui, pour cette raison, ne sont pas réductibles au résultat d'opérations formelles réalisées sur des représentations symboliques ou des propositions $^{66}$. Autrement dit, le travail de génération aussi bien que d'exploitation située des connaissances médicales doit avant tout être compris comme un processus de cognition distribuée, au sens d'E. Hutchins ${ }^{67}$.

Ces considérations, si elles s'avèrent légitimes, posent une limite de principe aux performances que les SBC exploitant des ontologies sont capables d'atteindre dans la production de connaissances médicales. On pourra bien entendu toujours affirmer qu'il est possible de développer des algorithmes capables de simuler la dynamique de la négociation et de l'argumentation humaine, qui permet de parvenir à ce consensus intersubjectif qu'est la connaissance. Mais on s'expose alors aux objections de Dreyfus qu'il est en principe impossible de formaliser les savoir-faire et connaissances présupposés par les performances des acteurs humains qui, par l'argumentation, sont capables de parvenir à un consensus. Un SBC pourra bien, sur la base d'une ontologie des EIM, extraire de bases de cas des couples potentiels d'effets indésirables et de médicaments. Mais il ne pourra jamais s'agir là que d'un système d'aide à l'élaboration de connaissances. Ce n'est toutefois un problème que si l'on se méprend sur le domaine de compétences des machines, en demandant à la cognition machinique de réaliser ce que, par principe, elle est incapable de faire, étant donné la nature formelle de son fonctionnement.

\section{Conclusion. Formalisation et représentation des connaissances}

Nous avons cherché à le montrer précédemment : seule une juste compréhension de ce que les machines ne peuvent faire, étant donné leur caractère machinique même, et qui reste, malgré sa finitude cognitive, une propriété exclusive de l'humain, peut offrir d'équilibrer convenablement la balance entre les tâches allouables aux machines et celles laissées à la charge de ce dernier, et permettre de développer des

Bouaud \& Séroussi (2005) prônent le développement de dispositifs qui préservent la liberté interprétative du médecin ainsi que son implication dans la décision médicale.

66. "Medical work, including the interpretation of signs and symptoms, is based as much on communication and negotiation between health care professionals and between professionals and patients, as on the cognitive thought processes of an individual physician." (Berg \& Toussaint, 2003, p. 226)

67. Cf. Hutchins (1995). 
dispositifs susceptibles d'assister efficacement le travail d'élaboration et d'exploitation des connaissances à l'œuvre dans le domaine médical ${ }^{68}$.

Nous avons notamment vu qu'en représentant les connaissances médicales dans des réseaux sémantiques (ontologies), on leur confère un caractère explicite et formel qui leur est manifestement étranger dans leur domaine effectif d'application. Mais est-ce véritablement problématique ? Après tout, comme nous le remarquions plus haut, la finalité de l'ingénierie des connaissances quand elle développe des ontologies n'est pas de modéliser le fonctionnement de la connaissance. Sa perspective n'est pas théorique, mais elle est opératoire : elle vise à concevoir des dispositifs capables de remplir certains attendus pratiques. Qu'elle soit contrainte de formaliser des connaissances qui ne possèdent pas une nature formelle dans le domaine où elles s'exercent importe donc peu, du moment que cette formalisation offre de développer des dispositifs capables d'assister efficacement l'activité humaine.

Une telle position est parfaitement défendable, et nous sommes tout disposés à y souscrire. Il convient toutefois à notre sens d'y apporter une série de précisions.

Tout d'abord, il n'est pas du tout certain que l'ingénierie des connaissances puisse développer des dispositifs performants pour assister l'activité concrète d'usage et de production de connaissances dès lors qu'elle exerce sur ces connaissances une transformation qui, pour employer une expression sans doute un peu forte, contrefait leur nature. En effet, conférer aux connaissances un caractère formel pour les rendre computables par des machines pourrait justement leur ôter ce qui les rend utilisables en situation par l'humain.

Par ailleurs, adopter une telle perspective n'est possible que si l'on renonce à l'optique représentationaliste qui se trouve généralement mise en avant dès lors qu'on prétend décrire la nature des ontologies et leur rapport à des connaissances qu'elles ont pour fonction, dit-on en effet fréquemment, de représenter. Comme l'explique Bachimont (2004), les ontologies «ne sont pas tant la représentation de connaissances que la médiation entre expressions de connaissance, permettant à l'utilisateur de naviguer et travailler sur des expressions dont il maîtrise l'interprétation. Elles sont donc des instruments intellectuels pour l'organisation de nos connaissances et non des modèles du monde. ${ }^{69}$. En recourant à une telle caractérisation, on court-circuite la question de l'adéquation au réel du formalisme que l'on adopte. La question n'est pas de savoir si la modélisation adoptée représente bien ou mal des connaissances existantes, si elle est fidèle à un domaine

68. Comme l'expliquent Dreyfus \& Dreyfus : "The debate of what computers should do is properly about social values and institutional forms. But before we can profitably discuss what computers should do we have to be clear about what they can do. We maintain that computers can't match human intuition and expertise, so in determining what computers should do we have to contrast their capacities with the more generous gifts possessed by the human mind." (Dreyfus \& Dreyfus, 1986, Préface, p. xi).

69. Bachimont (2004, p. 122). 
de connaissance donné (ou si les algorithmes de raisonnement implémentés dans les SBC modélisent correctement le raisonnement humain). Mais elle est de savoir si cette modélisation est capable d'assister l'activité cognitive individuelle et collective - et en particulier si elle permet de dépasser certaines limites inhérentes à la cognition humaine non prothétisée.

Ces dernières réflexions pourront paraître s'éloigner des problèmes traités dans le cadre de ce texte. Il n'en est rien. Si on admet que les ontologies ne représentent pas les connaissances d'un domaine, mais qu'elles constituent des artéfacts cognitifs offrant d'assister l'activité cognitive humaine, en l'aidant notamment à mieux s'orienter dans un milieu où la profusion de données nourrit l'illusion d'une disponibilité immédiate d'informations exploitables, les problèmes évoqués par Dreyfus tombent, pour une bonne part. Si on ne cherche pas à créer des machines qui pensent à notre place, des machines intelligentes, on n'est pas confronté au problème des prérequis non formalisables de l'intelligence humaine.

\section{Bibliographie}

Avillach P., Joubert M., Thiessard F., Trifirò G., Dufour J.C., Pariente A., Mougin F., Polimeni G., Catania M.A., Giaquinto C., Mazzaglia G., Fornari C., Herings R., Gini R., Hippisley-Cox J., Molokhia M., Pedersen L., Fourrier-Réglat A., Sturkenboom M., Fieschi M., "Design and evaluation of a semantic approach for the homogeneous identification of events in eight patient databases: a contribution to the European EUADR project", Studies in health technology and informatics, vol. 160 (2), 2010, p. 10851089.

Alecu I., Bousquet C., Degoulet P., Jaulent M.C., "PharmARTS: terminology web services for drug safety data coding and retrieval”, Medinfo, vol. 12 (1), 2007, p. 699-704.

Alecu I., Bousquet C., Jaulent M.C., "A case report: Using SNOMED CT for grouping adverse drug reactions terms", BMC Medical Informatics and Decision Making, vol. 8 (suppl 1), 2008, S4.

Bachimont B., Arts et Sciences du numérique : Ingénierie des connaissances et critique de la raison computationnelle, Mémoire d'habilitation à diriger des recherches, Université de Technologie de Compiègne, 2004.

Bachimont B., Nadah N., Baneyx A., Charlet J., "Categorial ontologies : Formalizing the differential ontology”, In D. Benslimane (coordinateur), $2^{e}$ Journées Francophones sur les Ontologies, Lyon, France, ACM, novembre 2008.

Bégaud B., Martin K., Haramburu F., Moore N., "Rates of spontaneous reporting of adverse drug reactions in France", Journal of the American Medical Association, vol. 288 (13), 2002, p. 1588.

Berg M., Toussaint T., "The mantra of modeling and the forgotten powers of paper: a sociotechnical view on the development of process-oriented ICT in health care", International Journal of Medical Informatics, vol. 69, 2003, p. 223-234. 
Bouaud J., Séroussi B., «OncoDoc : modélisation de bonnes pratiques thérapeutiques, mise en ouvre et évaluation », In R. Teulier, J. Charlet, P. Tchounikine, Ingénierie des connaissances, Paris, L'Harmattan, 2005, p. 229-250.

Bousquet C., Henegar C., Lillo-Le Louët A.L., Degoulet P., Jaulent M.C., "Implementation of automated signal generation in pharmacovigilance using a knowledge-based approach", International Journal of Medical Informatics, vol. 74 (7-8), 2005, p. 563-571.

Bousquet C., Trombert B., Kumar A., Rodrigues J.M., "Semantic categories and relations for modelling adverse drug reactions towards a categorial structure for pharmacovigilance", AMIA Annual Symposium Proceedings, 2008, p. 61-65.

Charlet J., Bachimont B., Mazuel L., Dhombres F., Jaulent M.C., Bouaud J., « OntoMenelas : motivation et retour d'expérience sur l'élaboration d'une ontologie noyau de la médecine », Journées Francophones des Ontologies, Poitiers, 2009.

Charlet J., L’Ingénierie des connaissances : développements, résultats et perspectives pour la gestion des connaissances, Mémoire d'habilitation à diriger des recherches, Université Pierre et Marie Curie, 2002.

Cimino J.J., "Collect once, use many. Enabling the reuse of clinical data through controlled terminologies", Journal of American Health Information Management Association, vol. 78 (2), 2007, p. 24-29.

Crevier D., À la recherche de l'intelligence artificielle, trad. N. Bucsek, Flammarion, 1997.

Douali N., Jaulent M.C., «Utilisation du web sémantique dans le raisonnement médical diagnostique. Domaine d'application: les infections des voies urinaires de l'adulte », Informatique et Santé, vol. 18, 2011, p. 59-70.

Dretske F., "Machines and the Mental", In S. Franchi, G. Guzeldere (eds.), Mechanical Bodies, Computational Minds. Artificial Intelligence from Automata to Cyborgs, M.I.T. Press, Cambridge, MA, 2005, p. 281-292.

Dreyfus H.L., «La portée philosophique du connexionnisme», In. D. Andler (dir.), Introduction aux sciences cognitives, 1992a, Paris, Gallimard, p. 352-373.

Dreyfus H.L., What computers still can't do. A critique of artificial reason, Cambridge/London : MIT Press, 1992 b.

Dreyfus H.L., Dreyfus S.E., Mind over machine: the power of human intuition and expertise in the era of the computer, Oxford, Basil Blackwell Ltd, 1986.

Gruber T., "A translation approach to portable ontology specifications", Knowledge Acquisition, vol. 5, 1993, p. 199-220.

Guarino N., "Undestanding, building, and using ontologies", International Journal of Human-Computer Studies, vol. 45 (2/3), 1997, p. 293-310.

Haugeland J., Artificial Intelligence: The Very Idea, Cambridge: MIT Press, 1985.

Heeks R., "Health information systems: Failure, success and improvisation", International Journal of Medical Informatics, vol. 75, 2006, p. 125-137. 
Henegar C., Bousquet C., Lillo-Le Louet A., Degoulet P., Jaulent M.C., "Building an ontology of adverse drug reactions for automated signal generation in pharmacovigilance", Computers in Biology and Medecine, vol. 36 (7-8), 2006, p. 748-767.

Hitzler P., Bader S., Garcez A., "Ontology learning as a use case for neural- symbolic integration", Proceedings of the IJCAI-05 Workshop on Neural-Symbolic Learning and Reasoning, NeSy'05, Edinburgh, UK, 2005.

Hutchins E., Cognition in the wild, MIT Press, 1995.

Jamet A., «Introduction à la Pharmacovigilance », Intervention orale au séminaire de recherche du groupe SPIM (Santé Publique et Informatique Médicale), INSERM, Paris, 10 janvier 2011.

Johnson M., The body in the mind: the bodily basis of meaning, imagination and reason, Chicago: The University of Chicago Press, 1987.

Kirsh D., “The Intelligent Use of Space”, Artificial Intelligence, vol. 73 (1-2), 1995, p. 31-68.

Lindsay P.H., Norman D.A., Traitement de l'information et comportement humain: une introduction à la psychologie, Montréal, Éditions Études Vivantes, 1980.

Mazuel L., Charlet J., «Alignement entre des ontologies de domaine et la Snomed : trois études de cas», in F. Gandon (Ed.), $20^{e}$ Journées Ingénierie des Connaissances, Hammamet, Tunisie, 2009, p. 1-12.

Mazuel L., Sabouret N., «Degré de relation sémantique dans une ontologie pour la commande en langue naturelle », Actes d'Ingénierie des Connaissances 2007 (IC2007), Grenoble, France, 2007, p. 73-85.

Meyboom R.H.B., Lindquist M., Egberts A.C.G., Edwards I.R., "Signal selection and followup in pharmacovigilance", Drug Safety, vol. 25 (6), 2002, p. 459-465.

Mohammed S.A., Sahroni N.S., "Information quality as success determinant for health information systems", ICT 2010 Regional Conference on Knowledge Integration, 2010, p. 674-679.

Nadkarni P.M., Darer J.A, "Determining correspondences between highfrequency MedDRA concepts and SNOMED: a case study", BMC Medical Informatics and Decision Making, vol. 10, 2010.

Newell A., Simon H.A., "Computer Science as Empirical Inquiry: Symbols and Search", Communications of the ACM, vol. 19 (3), 1976, p. 113-126.

Pisanelli D.M., Gangemi A., Massimo B., Catenacci C., "Coping with Medical Polysemy in the Semantic Web: the Role of Ontologies", MEDINFO 2004.

Putnam H. "The meaning of «meaning »", in Mind, Language and Reality, Cambridge, Cambridge University Press, 1975.

Rastier F., «Ontologie(s) », Revue des sciences et technologies de l'information, série : Revue d'Intelligence artificielle, vol. 18 (1), 2004, p. 15-40.

Rector A.L., Zanstra P.E., Solomon W.D., Rogers J.E., Baud R., "Reconciling users' needs and formal requirements: Issues in developing a re-usable ontology for medicine", IEEE Transactions on Information Technology in BioMedicine, vol. 2(4), 1999, p. 229-242. 
472 RSTI - RIA - 25/2011. Intelligence artificielle et santé

Rosch E., "Human categorization", In N. Warren (ed.), Advances in cross-cultural psychology, vol. 1, London: Academic Press, 1977.

Steiner P., «Cognitivisme et sciences cognitives », Labyrinthes, vol. 5, $\mathrm{N}^{\circ}$ spécial « Sciences cognitives et sciences sociales », 2005, p. 13-39.

Uschold M., Gruninger M., "Ontologies: principles, methods, and applications", Knowledge Engineering Review, vol. 11(2), 1996, p. 93-155. 